\title{
Observations of parametric subharmonic instability-induced near-inertial waves equatorward of the critical diurnal latitude
}

\author{
Xiao-Hui Xie, ${ }^{1,2}$ Xiao-Dong Shang, ${ }^{1}$ Hans van Haren, ${ }^{3}$ Gui-Ying Chen, ${ }^{1}$ \\ and Yuan-Zhi Zhang ${ }^{4}$ \\ Received 16 December 2010; revised 22 January 2011; accepted 28 January 2011; published 8 March 2011 .
}

[1] Moored current observations of 75 days duration in the northeastern South China Sea $\left(\sim 20^{\circ} \mathrm{N}\right)$ suggest that parametric subharmonic instability (PSI) of semidiurnal $\left(D_{2}\right)$ internal tides can not only generate waves of frequencies close to $D_{2} / 2$, but also excite near-inertial waves whose frequencies are different from $D_{2} / 2$. Time series of shear amplitudes clearly show a 14-day cycle. Although nearinertial and near-diurnal motions dominate the shear, this cycle is in phase with the fortnightly spring-neap cycle of $D_{2}$-waves. After separation of near-inertial and near-diurnal waves using band-pass filters, shear magnitudes for both motions still follow this 14-day cycle, rather than that of diurnal internal tides or variations of the local wind field. This strongly suggests that PSI equatorward of the critical latitude for $D_{2} / 2$ waves $\left(\sim 29^{\circ}\right)$ not only transfers $D_{2}$-energy to $D_{2} / 2$ waves, but also to high-mode near-inertial waves. Near-inertial waves $(f)$ and another subharmonic $\left(D_{2}-f\right)$, together with $D_{2}$ waves, compose a PSI-triad following strong interaction. Citation: Xie, X.-H., X.-D. Shang, H. van Haren, G.-Y. Chen, and Y.-Z. Zhang (2011), Observations of parametric subharmonic instability-induced near-inertial waves equatorward of the critical diurnal latitude, Geophys. Res. Lett., 38, L05603, doi:10.1029/2010GL046521.

\section{Introduction}

[2] Breaking internal waves induced by shear instabilities, which are mainly dependent on the presence of small vertical-scale (high-mode) motions, are the major sources of deep-sea mixing [Munk and Wunsch, 1998]. In the ocean interior, many observations [e.g., Pinkel, 1983; van Haren, 2007] suggested that the largest amplitudes of shear $S=$ $(\partial u / \partial z, \partial v / \partial z)$ are often induced by near-inertial waves (internal waves with frequency near the local inertial frequency, $f$ ). Atmospheric disturbances have long been known to be the dominant mechanism generating near-inertial waves near the sea surface. However, D'Asaro [1991] showed that most high-mode near-inertial waves, generating the highest shear values, have dissipated before they can enter the ocean interior and only low-mode can propagate a significant distance. Alternatively, parametric subharmonic instability

\footnotetext{
${ }^{1}$ Key Laboratory of Tropical Marine Environmental Dynamics. South China Sea Institute of Oceanology. Chinese Academy of Sciences, Guangzhou, China.

${ }^{2}$ Graduate School of Chinese Academy of Sciences, Beijing, China.

${ }^{3}$ Royal Netherlands Institute for Sea Research, Den Burg, Netherlands.

${ }^{4}$ Institute of Space and Earth Information Science. Chinese University of Hong Kong, Shatin, Hong Kong.

Copyright 2011 by the American Geophysical Union. 0094-8276/11/2010GL046521
}

(PSI), a nonlinear resonant triad interaction, can transfer energy of low-mode semidiurnal $\left(D_{2}\right)$ internal tides to highmode near-inertial waves [Hibiya et al., 2002; van Haren, 2005; Alford et al., 2007, Alford, 2008; Xie et al., 2008, 2009]. Nevertheless, the process was often thought to predominantly occur near the so-called diurnal critical latitudes $\left(\sim 29^{\circ}\right)$, where $f$ is equal to half the semidiurnal tidal frequency $\left(D_{2} / 2\right)$, because the most effective instability was assumed to happen for perturbations of frequency $D_{2} / 2$ [McComas and Bretherton, 1977; Staquet and Sommeria, 2002; MacKinnon and Winters, 2005; Gerkema et al., 2006]. Until recently, Korobov and Lamb [2008] used numerical model to show that PSI could generate subharmonics (daughter waves) whose frequencies were different from half the parent frequencies, and hence PSI-generated near-inertial waves are also expected to occur at non-critical latitudes.

[3] In this paper, moored current measurements in the northeastern South China Sea (SCS) are used to provide the first observational evidence that high-mode near-inertial waves are generated by PSI of semidiurnal internal tides equatorward of the critical diurnal latitude and suggest that PSI not only transfers $D_{2}$-energy to $D_{2} / 2$ waves, but also generates subharmonics whose frequencies are different from $D_{2} / 2$.

\section{Data}

[4] Ocean currents were evaluated from one mooring deployed at $118.41^{\circ} \mathrm{E}, 20.58^{\circ} \mathrm{N}$, where the water depth is $2474 \mathrm{~m}$ (Figure 1a) and internal tides are thought to mainly originate from Luzon Strait [Tian et al., 2003; Lien et al., 2005]. The mooring consisted of an upward-looking WHL75-I-Z ADCP at $\sim 450 \mathrm{~m}$ (data from 436 to $52 \mathrm{~m}, 16-\mathrm{m}$ bins) and five Aanderaa current meters at 800-, 1000-, 1500-, 2000-, and 2300-m. In this letter, only ADCP data are used. The mooring period was from August 2000 to March 2001. Unfortunately, the ADCP worked only for the first $\sim 75$ days (from the 20th of August to 4th of November, 2000). Its sampling interval was 15 minutes.

[5] In order to investigate the influence of wind on nearinertial waves, the current evolution at mooring location is simulated using a one-dimensional mixed layer model, Price-Weller-Pinkel (PWP) [Price et al., 1986]. The wind field and temperature-salinity profile used in the model are from the swath Quick Scatterometer (QuikSCAT) data and the World Ocean Atlas 2001, respectively.

\section{Results}

\subsection{Spectral Analysis}

[6] Since kinetic energy spectra and 16-m current difference spectra at most depths observed by the ADCP showed 

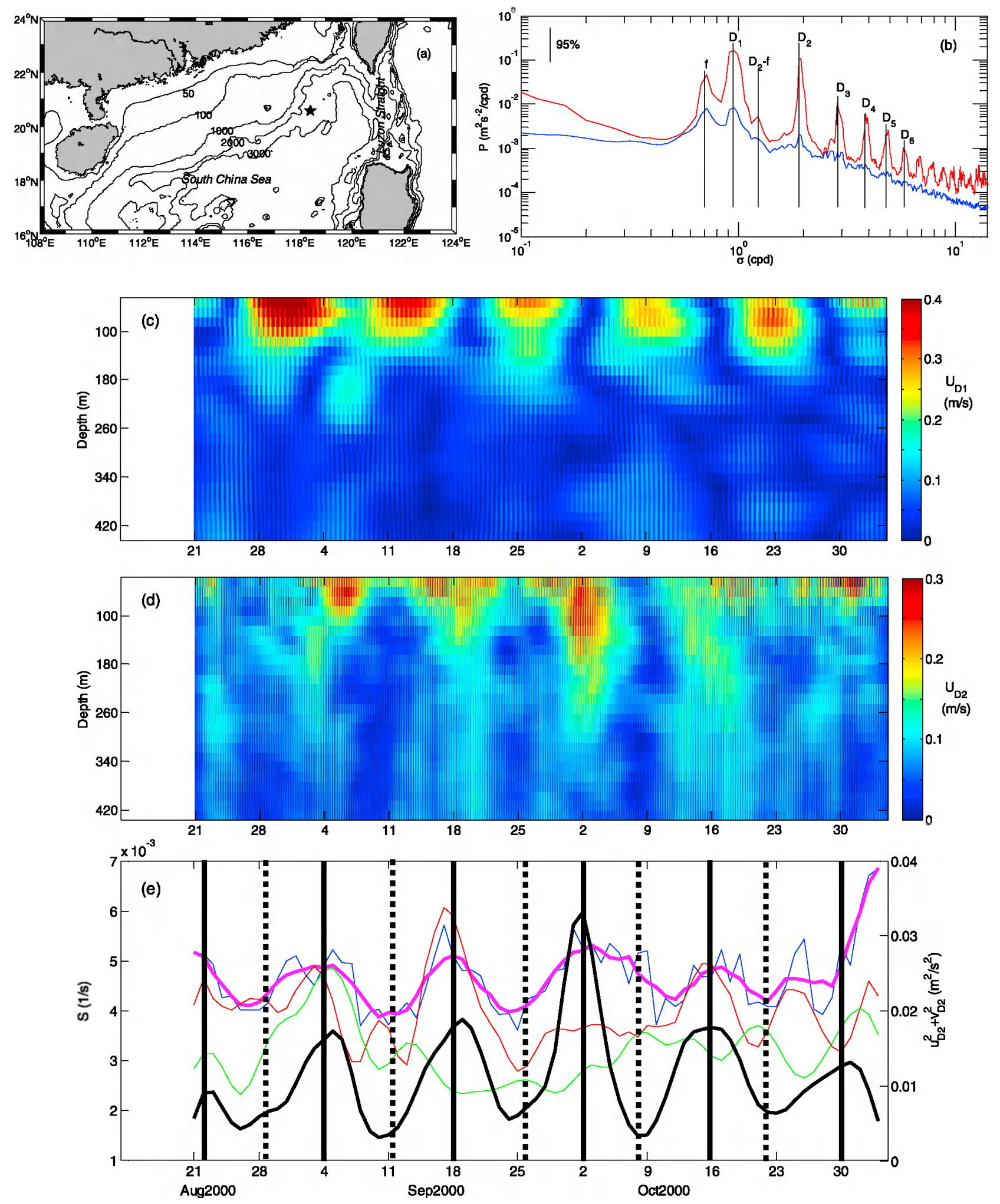

Figure 1. (a) Mooring position (star) in the northeastern South China Sea. (b) Kinetic energy (red) and 16-m current difference (blue) spectra averaged over 52 to $324 \mathrm{~m}$. The long vertical lines represent various frequencies $\left(f, D_{1}, \ldots\right)$. The short vertical bar in the upper-left corner indicates the $95 \%$ statistical significance level. Depth-time series of band-pass filtered (c) $D_{1}$ and (d) $D_{2}$ velocity amplitudes. Note different color scales. (e) Time series of 24 -hour mean semidiurnal kinetic energy (black) and raw $\left(S\right.$, blue), inertial $\left(2 \times S_{f}\right.$, red) and diurnal $\left(2 \times S_{D 1}\right.$, green) shear amplitudes averaged over 52 to $324 \mathrm{~m}$. Magenta curve is a 5-point moving average of the raw shear (blue curve). The filter bounds are [0.85, 1.15] $f$ for near-inertial motions, $\left[0.9 O_{1}, 1.1 K_{1}\right]$ for $D_{1}$ and $[0.9,1.1] M_{2}$ for $D_{2}$. The solid and dotted vertical bars in Figure 1e indicate semidiurnal spring (diurnal neap) and diurnal spring (semidiurnal neap) tides, respectively. Similar treatments are also applied to Figures $2 b, 2 c$, and $3 a$ below. 
similar patterns, they are averaged vertically over the depth range 52 to $324 \mathrm{~m}$. The average spectra are given in Figure $1 \mathrm{~b}$. From the kinetic energy spectra, we can observe that the most obvious peaks appear in inertial $(f)$, diurnal $\left(D_{1}\right)$ and semidiurnal $\left(D_{2}\right)$ frequency bands. Hereafter, we use $D_{i}, \mathrm{i}=$ $1,2,3$, etc. to represent an (over-) diurnal frequency. A rawer kinetic energy spectrum than displayed in Figure $1 \mathrm{~b}$ shows that diurnal $O_{1}$ and $K_{1}$ are dominant in $D_{1}$-band and that $D_{2}$-band is borne primarily by semidiurnal $M_{2}$. The inertial peak is clearly distinguished from $D_{1}$-peak. In addition to these fundamental inertial and tidal frequency bands, significant peaks were also found at some higher tidal harmonic bands (e.g., $D_{3}, D_{4}$ ). In 16-m current difference spectra, the strongest signal occurred at $f$ and $D_{1}$. Although $D_{2}$ has strong signal in velocity, it is rather weak in shear, indicating that semidiurnal energy is dominated by low-mode. In addition, whether it is in kinetic energy spectra or 16-m current difference spectra, a relatively weak peak is observed at a difference interaction frequency between $f$ and $D_{2}$, i.e., $D_{2}-f$.

\subsection{Time Series}

[7] Taking advantage of spectral gaps between different peaks, a fourth-order Butterworth filter was designed to detach these peak signals. Figures $1 \mathrm{c}$ and $1 \mathrm{~d}$ show depthtime series of band-pass filtered diurnal and semidiurnal velocity amplitudes $U_{D 1}$ and $U_{D 2}$, respectively. The large velocity amplitudes for both tidal motions are often found in a narrow depth range near the surface, implying the dominance of internal tides over barotropic tides. In the meantime, the relatively large $U_{D 1}$ and $U_{D 2}$ values in the upper $180 \mathrm{~m}$ show a clear, fortnightly spring-neap cycle. However, the diurnal and semidiurnal 14-cycles are nearly out of phase. Note that the diurnal 14-day cycle becomes unclear below $200 \mathrm{~m}$. This is because $O_{1}$ and $K_{1}$ become relatively weak at these depths and peaks in $D_{1}$-band also appear at some non-tidal frequencies (e.g., $M_{1}=M_{2} / 2$ ) (not shown).

[8] Figure 1e shows time-series of the depth-average raw $(S)$, inertial $\left(S_{f}\right)$ and diurnal $\left(S_{D 1}\right)$ shear amplitudes. Semidiurnal kinetic energy $\left(u_{D 2}^{2}+v_{D 2}^{2}\right)$ is also shown in Figure 1e. Both enhanced near-inertial and diurnal shears often occur at semidiurnal spring tide. As a result, due to the fact that $S$ is dominated by near-inertial and diurnal frequencies, its elevation follows semidiurnal spring tide for most observation periods, rather than diurnal spring tide. The correlation coefficient between semidiurnal kinetic energy (Figure le, black line) and shear variance (Figure 1e, magenta line) from August 21 st to October 31 st is 0.67 , markedly exceeding the $99 \%$ significance level $(=0.3)$. Similar results can also be found at individual depth bins (see Figure $2 \mathrm{~d}$ below). These suggest that high-mode near-inertial and near-diurnal waves are strongly linked to semidiurnal internal waves.

[9] To confirm further that both $f$ and $D_{1}$ motions are associated with semidiurnal internal tides, we examine depthtime series of their band-pass filtered $S_{f}$ and $S_{D 1}$. From Figures $2 \mathrm{~b}$ and $2 \mathrm{c}$, one can again find that both large $S_{f}$ and $S_{D 1}$ often occur at semidiurnal spring tide. The heightened $S_{f}$ around a depth of $180 \mathrm{~m}$ for the entire observation period closely follows the semidiurnal spring-neap cycle. Near this depth, as well as around $260 \mathrm{~m}$, the correlation coefficients for $S_{f}$ and semidiurnal kinetic energy are above the $99 \%$ significance level (Figure 2d). The clear 14-day modulation rules out the possibility that the enhanced near-inertial sig- nals at semidiurnal spring tide are caused by wind because the local wind speed and wind-generated near-inertial waves in the mixed layer do not show a 14-day cycle (Figure 2a). However, the wind field also has notable effects on high-mode near-inertial signals. For example, nearinertial shear is enhanced around October 9th, 2000 near the surface (upper $160 \mathrm{~m}$ ) when the wind speeds are markedly increased (Figures $2 \mathrm{a}$ and 2b). But the sub-surface (below $160 \mathrm{~m}$ ) shear seems not to be influenced, implying that wind may not be the direct cause of large near-inertial shear in the ocean interior. As to $D_{1}$ motions, the diurnal 14-day cycle is inconspicuous in the shear field (Figure 2c). Large $U_{d}$ during diurnal spring tide does not noticeably elevate shear, suggesting the dominance of low-mode diurnal internal tidal waves. The clearest evidence that energy of highmode diurnal waves during semidiurnal springs is mainly supplied by $D_{2}$ waves appears near $324 \mathrm{~m}$ depth, where the elevated $S_{D 1}$ mostly occurs at semidiurnal spring (diurnal neap) tide (Figure $2 \mathrm{c}$ ) and the correlation coefficient between two time series of $S_{D 1}$ and semidiurnal kinetic energy is also above the 99\% significance level (Figure 2d).

\section{Discussion}

[10] In the above observations, we showed that both near-inertial and near-diurnal shears follow a 14-day cycle that is consistent with semidiurnal spring-neap. This suggests that energy of the intensified high-mode near-inertial and near-diurnal waves at semidiurnal spring tide is mainly provided by waves of frequency $D_{2}$. The enhanced $D_{1}$ shear associated with $D_{2}$ waves can be attributed to PSI mechanism, as energy is transferred from low-mode waves with frequency $D_{2}$ to high-mode waves with frequency $D_{2} / 2=D_{1}$. Nevertheless, the energy transfer from $D_{2}$ waves to nearinertial waves seems to be different from the classical PSI course, as $f$ is significantly unequal to $D_{2} / 2$. Using an inviscid two-dimensional numerical model, Korobov and Lamb [2008] suggested that when PSI turned into a strongly nonlinear instability from a weakly nonlinear instability, it could generate subharmonics whose frequencies were different from half the original frequencies. Thus, the enhanced near-inertial waves associated with semidiurnal internal tides here may be the result of strongly nonlinear instabilities. However, a PSI-triad requires a third wave-packet of frequency $D_{2}-f$, according to the frequency relation in PSItheory [McComas and Bretherton, 1977], namely $\omega_{0}=\omega_{1}+$ $\omega_{2}$, where $\omega_{0}$ is frequency of parent wave and $\omega_{1}$ and $\omega_{2}$ are frequencies of two daughter waves.

[11] At depths of 100 and $196 \mathrm{~m}$, the velocity amplitudes $U_{D 2-f}$ clearly display a 14 -day cycle (Figure $3 \mathrm{a}$ ). This cycle is also in phase with the semidiurnal spring-neap cycle. Examination of the phase $\phi=\tan ^{-1} v_{D 2-f} / u_{D 2-f}$ (Figure $3 b$ ), reveals a strong clockwise rotation $(\partial \phi / \partial t<0)$, excluding cycle-jumps. This indicates that the strengthened $D_{2}-f$ waves is not caused by fine-structure contamination [Alford, 2001]. Figure $3 \mathrm{~b}$ also demonstrates an approximate $180^{\circ}$ phase difference between velocity signals of $D_{2}-f$ motions at depths of 100 and $196 \mathrm{~m}$. These waves have a high vertical wavenumber. These properties suggest that $D_{2}-f$ waves are generated via PSI of semidiurnal internal waves, i.e., waves of frequency $D_{2}, f$ and $D_{2}-f$ for a PSI-triad. Depth-time maps of band-passed filtered $f$ and $D_{2}-f$ zonal velocities ( $u_{f}$ and $u_{D 2-f}$ ) during the third semidiurnal spring tide indi- 

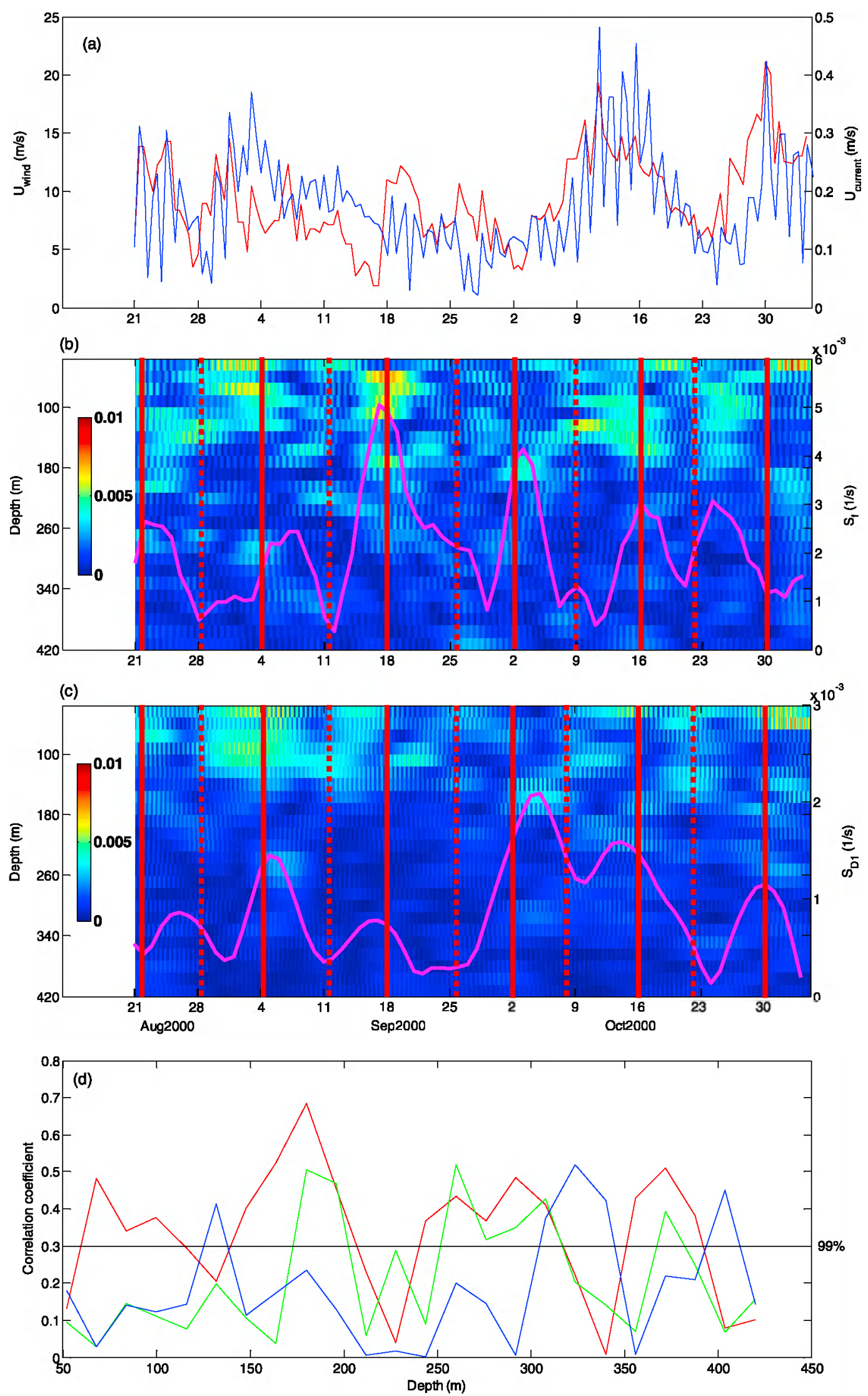

Figure 2. (a) Time series of wind (red) and current (blue) speeds from PWP model. (b) Depth-time series of observed $S_{f}$. (c) Depth-time series of observed $S_{D 1}$. Time series of $S_{f}$ at $180 \mathrm{~m}$ and $S_{D 1}$ at $324 \mathrm{~m}$ are superimposed on the corresponding depth-time map, respectively. (d) Absolute value of correlation coefficients between semidiurnal kinetic energy and shear variance including $S$ (red), $S_{f}$ (green) and $S_{D 1}$ (blue). The horizontal line gives the $99 \%$ significance level. 

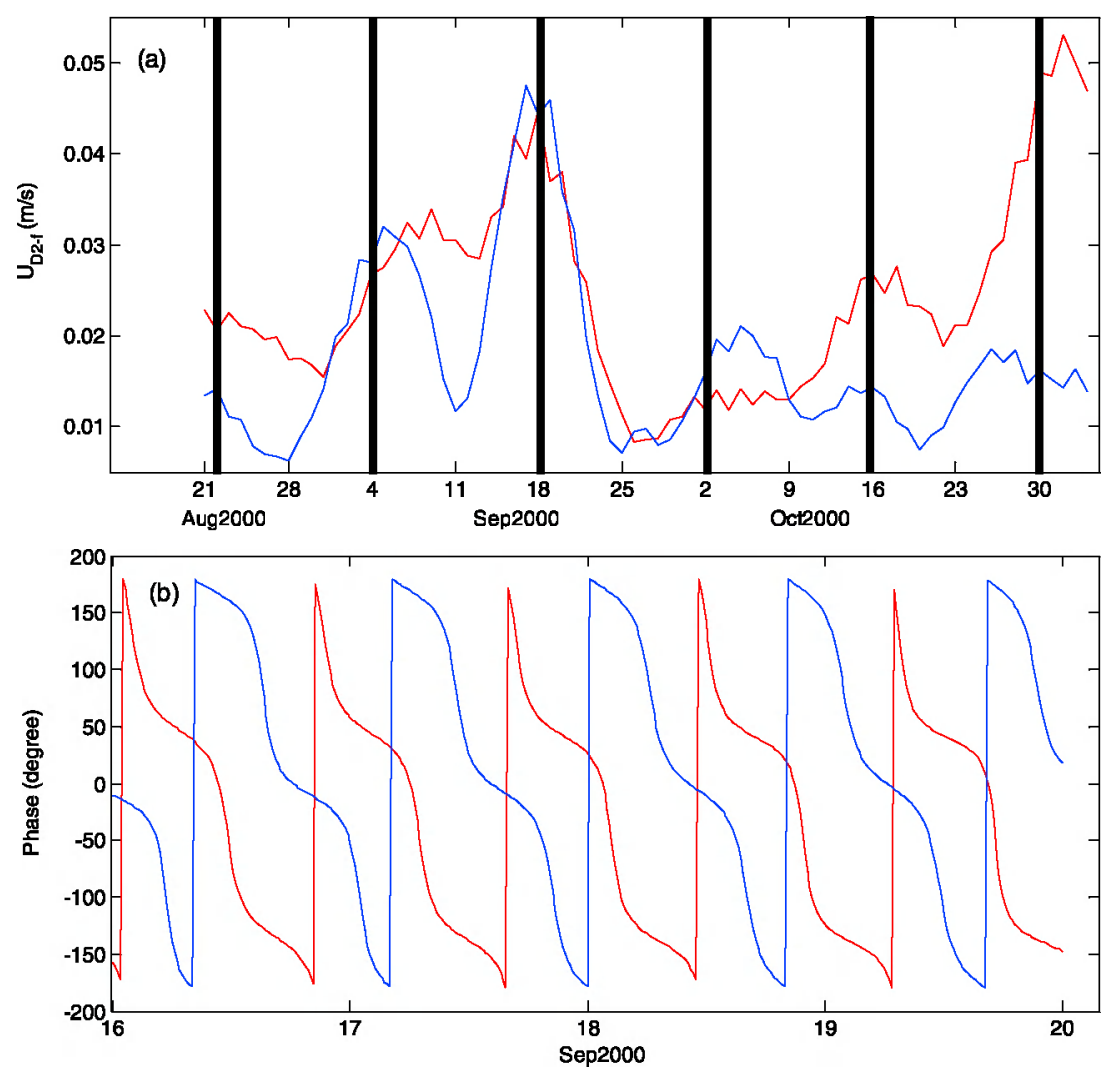

(c)

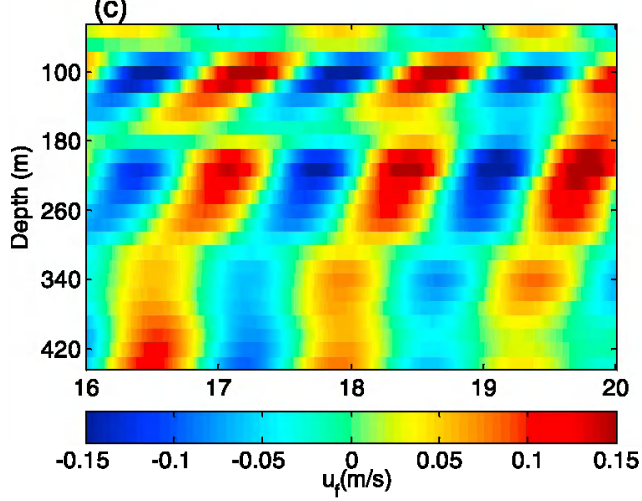

(d)

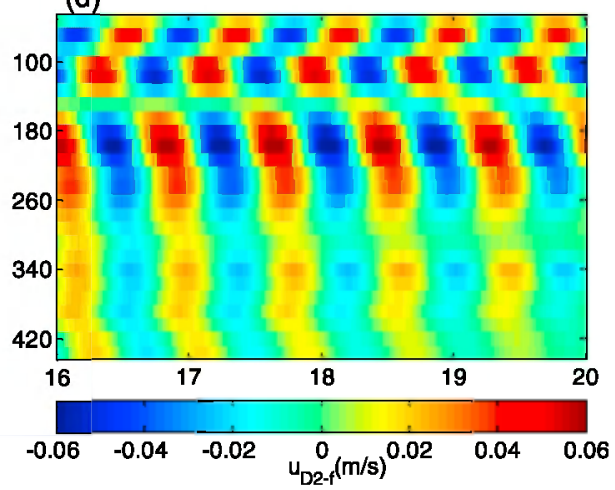

Figure 3. (a) Time series of 24-hour-averaged $U_{D 2 f}$ at 100 (red) and $196 \mathrm{~m}$ (blue). The filter bound is $[0.9,1.1]\left(M_{2}-f\right)$. (b) Phase variations of $D_{2}-f$ waves during the third semidiurnal spring tide at depths of 100 (red) and $196 \mathrm{~m}$ (blue). Depthtime maps of (c) $u_{f}$ and (d) $u_{D 2-f}$ from September 16 to 20 .

cate that two daughter waves in the PSI-triad of $D_{2}=f+$ $D_{2}-f$ have opposite (high) vertical wavenumbers (Figures $3 \mathrm{c}$ and 3d), also agreeing well with PSI-theory [McComas and Bretherton, 1977]. Near $180 \mathrm{~m}$ depth, both wave-packets show an abrupt phase change. Due to the fact that the vertical wavenumber of waves is easily affected by stratification, the pattern may be associated with the depth variation of buoyancy frequency. In addition, vertically-standing wave signals are often observed in diurnal velocity and shear fields during semidiurnal spring tides (not shown). These signals can be attributed to the superposition of two daughter waves with nearly opposite vertical wavenumbers and nearly equal frequencies in the PSI-triad of $D_{2}=D_{1}+D_{1}$.

[12] In order to further provide evidence of PSI, bicoherence spectrum (normalized bispectrum) is explored. This can be used to distinguish the extent of quadratic phase coupling in a deterministic signal from independently excited waves [van Haren, 2005]. Since PSI of $D_{2}$ waves often occurs at semidiurnal spring tide, we pick out six sixday long (Hanning-tapered) time series from six semidiurnal springs to compute bicoherence, yielding a spectral estimation with $\sim 15$ degrees of freedom.

[13] Figure 4 shows averaged bicoherence of $u$ and $v$ velocity components. Bicoherence analysis at $148 \mathrm{~m}$ shows that a significant bicoherence value occurs around $[0.83$, 1.17] cpd (Figure 4a), which is further evidence for the occurrence of semidiurnal internal tidal PSI. Significant bicoherence also appears around [2,2] cpd, suggesting that waves with frequency $D_{4}$ is mainly generated by selfnonlinear interaction of $D_{2}$ waves, namely $D_{2}+D_{2}=D_{4}$, 

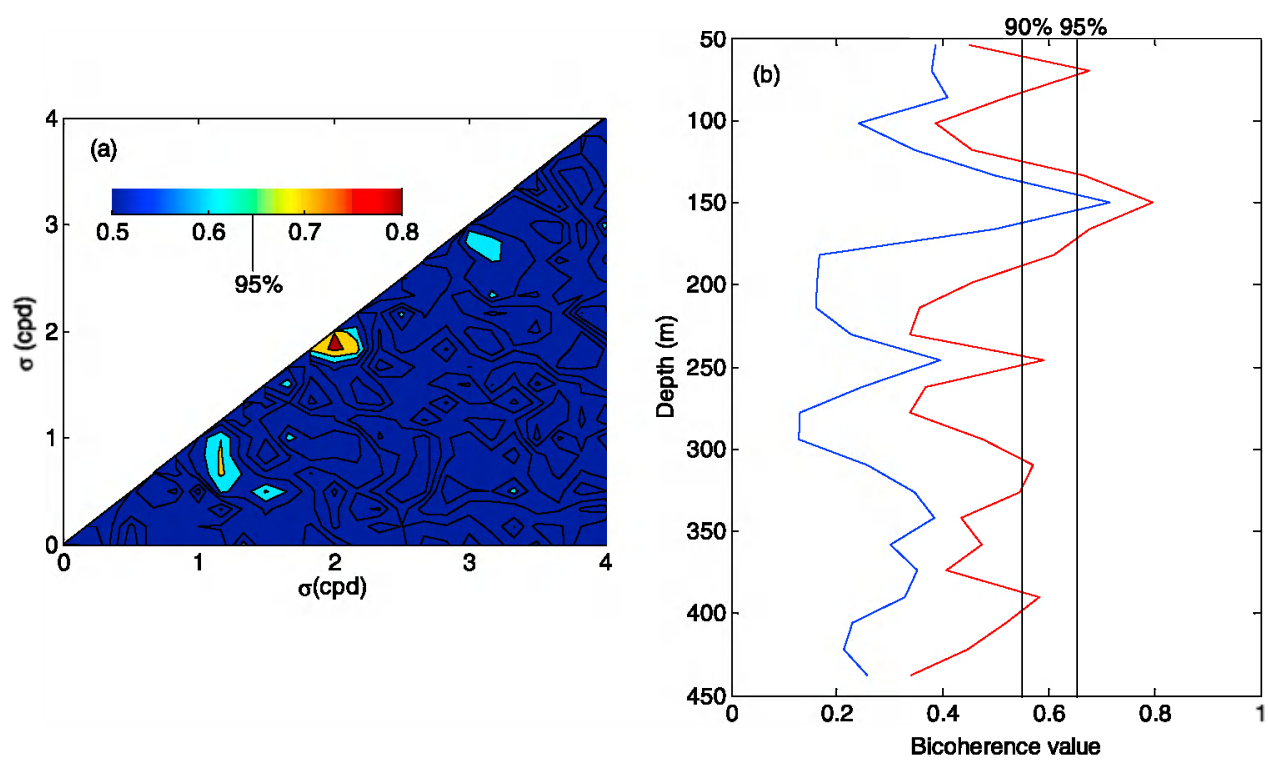

Figure 4. (a) Average bicoherence of $u$ and $v$ at $148 \mathrm{~m}$. Note that all values less than 0.5 are set to 0.5 for clarity. (b) Bicoherence values around $[0.83,1.17]$ cpd (blue) and $[2,2]$ cpd (red) plotted as a function of depth. The vertical lines give $90 \%$ and $95 \%$ significance levels.

rather than by Doppler shifting. For subharmonic interaction of $D_{2}$ waves, bicoherence analysis of all observed depths indicate that significant results at the $90 \%$ level only appear over a limited range of vertical depths (Figure 4b), being similar to observations by van Haren [2005] and Carter and Gregg [2006]. However, bicoherence values around [0.83, 1.17] cpd reveal a similar depth variation with that around $[2,2]$ cpd (Figure 4b), implying that semidiurnal internal tidal PSI, like $D_{2}+D_{2}=D_{4}$, may also occur at other depths.

\section{Summary and Conclusion}

[14] Current profiles from a set of $\sim 75$-day long moored ADCP in the northeastern SCS were used to demonstrate that PSI of internal tides can generate subharmonics whose frequencies are different from half the internal tidal frequency. In addition to near-diurnal internal waves $\left(D_{2} / 2\right)$, PSI also effectively transfer energy of low-mode semidiurnal internal tides to high-mode near-inertial waves for which $f \neq D_{2} / 2$. This is the first time that PSI-induced near-inertial waves have been found in the field observations equatorward of the critical latitude. This phenomenon is important because PSI presented here is somewhat different from the weak resonance theory provided by McComas and Bretherton [1977]. Furthermore, it suggests that the influence of internal tidal PSI on large near-inertial shears in the ocean interior does not only occur at the critical latitude. Due to the fact that strong internal tides in the observation region can propagate as a narrow tidal beam [Lien et al., 2005], strongly nonlinear instability may be a main reason of the enhanced high-mode near-inertial waves [Carter and Gregg, 2006; Korobov and Lamb, 2008]. Future studies should reconsider some concepts of PSI-theory to explore the effectiveness of various waves in the subharmonic instability of parent waves.

[15] The occurrence of PSI for the entire 75-day is not occasional, as the shear amplitude is heightened at each semidiurnal spring tide. This also implies a rapid, persistent energy transfer to PSI-triads. Clearly, PSI is one of domi- nant mechanisms of the cascade of internal tidal energy down to small dissipation scales available for ocean mixing.

[16] Acknowledgments. We thank two anonymous reviewers for their valuable comments. We also thank D. -X. Wang for the data used in this study. This work is supported by projects $\mathrm{KZCX1-YW-12-01,}$ $\mathrm{U} 1033002,40976010$ and $2011 \mathrm{CB} 403505$.

[17] Meric A. Srokosz thanks two anonymous reviewers.

\section{References}

Alford, M. H. (2001), Fine-structure contamination: Observations and a model of a simple two-wave case, J. Phys. Oceanogr., 31, 2645-2649, doi: $10.1175 / 1520-0485(2001) 031<2645$ :FSCOAA $>2.0 . \mathrm{CO} ; 2$

Alford, M. H. (2008), Observations of parametric subharmonic instability of the diurnal internal tide in the South China Sea, Geophys. Res. Lett., 35, L15602, doi:10.1029/2008GL034720.

Alford, M. H., J. A. MacKinnon, Z. Zhao, R. Pinkel, J. Klymak, and T. Peacock (2007), Internal waves across the Pacific, Geophys. Res. Lett., 34, L24601, doi:10.1029/2007GL031566.

Carter, G. S., and M. C. Gregg (2006), Persistent near-diurnal internal waves observed above a site of $M_{2}$ barotropic-to-baroclinic conversion, J. Phys. Oceanogr., 36, 1136-1147, doi:10.1175/JPO2884.1.

D'Asaro, E. A. (1991), A strategy for investigating and modeling internal wave sources and sinks, in Dynamics of Oceanic Internal Gravity Waves II: Proceedings of 'Aha Huliko'a Hawaiian Winter Workshop, pp. 451-466, Univ. of Hawaii at Manoa, Honolulu.

Gerkema, T., C. Staquet, and P. Bouruet-Aubertot (2006), Decay of semidiurnal internal-tide beams due to subharmonic resonance, Geophys. Res. Lett., 33, L08604, doi:10.1029/2005GL025105.

Hibiya, T., M. Nagasawa, and Y. Niwa (2002), Nonlinear energy transfer within the oceanic internal wave spectrum at mid and high latitudes, J. Geophys. Res., 107(C11), 3207, doi:10.1029/2001JC001210.

Korobov, S. A., and K. Lamb (2008), Interharmonics in internal gravity waves generated by tide-topography interaction, J. Fluid Mech., 611, 61-95, doi:10.1017/S0022112008002449.

Lien, R.-C., T. Y. Tang, M. H. Chang, and E. A. D'Asaro (2005), Energy of nonlinear internal waves in the South China Sea, Geophys. Res. Lett. 32, L05615, doi:10.1029/2004GL022012.

MacKinnon, J. A., and K. B. Winters (2005), Subtropical catastrophe: Significant loss of low-mode tidal energy at $28.9^{\circ} \mathrm{N}$, Geophys. Res. Lett., 32 , L15605, doi:10.1029/2005GL023376.

McComas, C. H., and F. P. Bretherton (1977), Resonant interaction of oceanic internal waves, J. Geophys. Res., 82(9), 1397-1412, doi:10.1029/ JC082i009p01397. 
Munk, W., and C. Wunsch (1998), Abyssal recipes. II: Energetics of tidal and wind mixing, Deep Sea Res., Part I, 45, 1977-2010, doi:10.1016/ S0967-0637(98)00070-3.

Pinkel, R. (1983), Doppler sonar observations of internal waves: Wavefield structure, J. Phys. Oceanogr., 13, 804-815, doi:10.1175/15200485 (1983)013<0804:DSOOIW $>2.0 . \mathrm{CO} ; 2$.

Price, J. F., R. A. Weller, and R. Pinkel (1986), Diurnal cycling: Observations and models of the upper ocean response to diurnal heating, cooling, and wind mixing, J. Geophys. Res., 91, 8411-8427, doi:10.1029/ JC091iC07p08411.

Staquet, C, and J. Sommeria (2002), Internal gravity waves: From instabilities to turbulence, Annu. Rev. Fluid Mech., 34, 559-593, doi:10.1146 annurev fluid. 34.090601.130953.

Tian, J., L. Zhou, X. Zhang, X. Liang, Q. Zheng, and W. Zhao (2003), Estimates of $M_{2}$ internal tide energy fluxes along the margin of northwestern Pacific using TOPEX/POSEIDON altimeter data, Geophys. Res. Lett., 30(17), 1889, doi:10.1029/2003GL018008.

van Haren, H. (2005), Tidal and near-inertial peak variations around the diurnal critical latitude, Geophys. Res. Lett., 32, L23611, doi:10.1029/ 2005GL024160. van Haren, H. (2007), Shear at the critical diurnal latitude, Geophys. Res Lett., 34, L06601, doi:10.1029/2006GL028716.

Xie, X.-H., G.-Y. Chen, X.-D. Shang, and W.-D. Fang (2008), Evolution of the semidiurnal $\left(M_{2}\right)$ internal tide on the continental slope of the northern South China Sea, Geophys. Res. Lett., 35, L13604, doi:10.1029/ 2008GL034179.

Xie, X.-H., X.-D. Shang, G.-Y. Chen, and L. Sun (2009), Variations of diurnal and inertial spectral peaks near the bi-diurnal critical latitude, Geophys. Res. Lett., 36, L02606, doi:10.1029/2008GL036383.

G.-Y. Chen, X.-D. Shang, and X.-H. Xie, Key Laboratory of Tropical Marine Environmental Dynamics, South China Sea Institute of Oceanology, Chinese Academy of Sciences, Guangzhou 510301, China. (xdshang( scsio.ac.cn)

H. van Haren, Royal Netherlands Institute for Sea Research, PO Box 59 NL-1790 AB Den Burg, Netherlands.

Y.-Z. Zhang, Institute of Space and Earth Information Science, Chinese University of Hong Kong, Fok Ying Tung Remote Sensing Science Building, Shatin, New Territories, Hong Kong. 